\title{
Positively curved 6-manifolds with simple symmetry groups
}

\author{
FUQUAN FANG \\ Nankai Institute of Mathematics, Nankai University, Tianjin 300071, P.R.C. \\ Instituto de Matemática, Universidade Federal Fluminense, Niterói, RJ, Brasil \\ Manuscript received on July 16, 2002; accepted for publication on September 3, 2002; \\ presented by MANFREDO DO CARMO
}

\begin{abstract}
Let $M$ be a simply connected compact 6-manifold of positive sectional curvature. If the identity component of the isometry group contains a simple Lie subgroup, we prove that $M$ is diffeomorphic to one of the five manifolds listed in Theorem A.
\end{abstract}

Key words: 6-manifolds, positive curvature, symmetry groups.

\section{INTRODUCTION}

An interesting problem in Riemannian geometry is to classify positively curved manifolds whose isometry groups are large. A typical example is the classification of homogeneous manifolds of positive curvature (cf. Aloff and Wallach 1975, Berger 1961, Bergery 1976, Grove and Searle 1997).

Recently, there has been considerable progress on classification of positively curved manifolds with large abelian symmetry group actions (cf. Hsiang and Kleiner 1989, Fang and Rong 2001, 2002, Grove and Searle 1994, Rong 2002, Wilking 2002). The maximal rank theorem of GroveSearle asserts that if a compact positively curved manifold $M$ admits an isometric $T^{k}$-action, then $k \leq\left[\frac{n+1}{2}\right]$ and " $=$ ", if and only if $M$ is diffeomorphic to a sphere, a lens space or a complex projective space (Grove and Searle 1994). The homeomorphism classification for positively curved $n$-manifolds with almost maximal rank have been carried out for $n=4$ by Hsiang and Kleiner (1989), for $n=5$ by Rong (2002) and for $n \geq 8$ completely solved by Fang and Rong (2001). Very recently Wilking (2002) announced the following 1/2-maximal rank theorem: For $n \geq 10$, if a closed simply connected positively curved $n$-manifold $M$ with symmetry rank (i.e. the rank of its isometry group) at least $\frac{n}{4}+1$, then $M$ is homeomorphic to a sphere or a quaternionic projective space or homotopic to a complex projective space (Wilking 2002).

E-mail: ffang@nankai.edu.cn / fuquan@mat.uff.br 
In the geometry of positive sectional curvature, 6-dimensional manifolds play very interesting roles. This is because, there are so called "flag" manifolds, the homogeneous space $S U(2) / T^{2}$ and the biquotient space $S U(3) / / T^{2}$ on which there exist infinitely many circle bundles whose total spaces (dimension 7) admit positive sectional curvature, the Aloff-Wallach spaces and the Eschenberg spaces (cf. Aloff and Wallach 1975, Eschenberg 1982, 1992). Except the two flag manifolds, so far only $S^{6}$ and $\mathbb{C P}^{3}$ are known to admit positive curvature metrics. All of the four examples admit a symmetry of a simple Lie group.

The purpose of this paper is to study the isometric $G$-actions on positively curved manifolds of dimension 6 where $G$ is a simple Lie group. We will prove almost a converse holds, except that one more topology type, $S^{2} \times S^{4}$, maybe occur.

In the below $=$ will indicate manifolds of the same diffeomorphism type.

THEOREM A. Let $M$ be a simply connected compact 6-manifold of positive sectional curvature. If the identity component of the isometry group of $M$ contains a simple Lie subgroup. Then $M=S^{6}, S^{2} \times S^{4}, \mathbb{C P}^{3}, S U(3) / T^{2}$ or $S U(3) / / T^{2}$.

Note that any simple Lie group contains either $S U(2)$ or $S O(3)$ as a subgroup. Therefore one really needs only to study manifolds with symmetry group $G=S U(2)$ or $G=S O(3)$. Thus Theorem A follows immediately from the following two results.

THEOREM B. Let $M$ be a simply connected compact 6-manifold of positive sectional curvature. If $M$ admits an effective isometric $S U(2)$-action, then $M=S^{6}, S^{2} \times S^{4}, \mathbb{C P}^{3}, S U(3) / T^{2}$ or $S U(3) / / T^{2}$.

THEOREM C. Let $M$ be a simply connected compact 6-manifold of positive sectional curvature. If $M$ admits an isometric $S O(3)$-action (not necessarily effective) then $M=S^{6}, S^{2} \times S^{4}, \mathbb{C P}^{3}$.

The rest of the paper is organized as follows: In Section 1, we collect some necessary preliminaries in the proof of Theorems B and C. In Section 2, we prove Theorems B and C.

\section{PRELIMINARIES}

\section{A. Positive Curvature}

For positively curved simply connected manifolds, few general results are known. In our proof of Theorem A, the following results in this subsection are required.

Theorem 1.1 (Synge 1936). Let $M$ be an $n$-manifold of $\sec _{M}>0$.

(1.1.1) If $n$ is even and $M$ is orientable, then any orientation preserving isometry $\phi$ has a non-empty fixed point set.

(1.1.2) If $n$ is odd, then $M$ is orientable and any orientation reversing isometry $\phi$ has a non-empty fixed point set. 
THEOREM 1.2 (FRANKel 1961). Let M be a compact manifold of positive sectional curvature. If two totally geodesic submanifolds $N_{1}$ and $N_{2}$ satisfies

$$
\operatorname{dim}\left(N_{1}\right)+\operatorname{dim}\left(N_{2}\right) \geq \operatorname{dim}(M)
$$

then $N_{1}$ and $N_{2}$ have a non-empty intersection.

The asymptotic index of an immersion $f: N \rightarrow M$ is defined by $v_{f}=\min _{x \in N} v_{f}(x)$, where $v_{f}(x)$ is the maximal dimension of a subspace of $T_{x} N$ on which the second fundamental form vanishes. Clearly, $f$ is a totally geodesic immersion if and only if $v_{f}=\operatorname{dim}(N)$.

THEOREM 1.3 (FANG ET AL. 2002). Let $M^{m}$ be a closed manifold of positive sectional curvature, and let $N_{j} \hookrightarrow M^{m}$ be a closed embedded submanifold of asymptotic index $v_{j}(j=1,2)$. If either $N_{j}(j=1,2)$ is minimal or $N_{1}=N_{2}$, then the following natural homeomorphisms,

$$
\pi_{i}\left(N_{1}, N_{1} \cap N_{2}\right) \rightarrow \pi_{i}\left(M^{m}, N_{2}\right), \quad \pi_{i}\left(N_{2}, N_{1} \cap N_{2}\right) \rightarrow \pi_{i}\left(M^{m}, N_{1}\right),
$$

are isomorphism for $i \leq v_{1}+v_{2}-m$ and are surjections for $i=v_{1}+v_{2}-m+1$.

Applying to totally geodesic submanifold, where the asymptotic index is exactly the dimension, Theorem 1.3 implies immediately that

THEOREM 1.4 (WILKING 2002). If $M^{n}$ has positive sectional curvature and if $N^{n-k}$ is an embedded totally geodesic submanifold then the inclusion map $i: N^{n-k} \rightarrow M^{n}$ is $n-2 k+1$ connected, that is $i$ induces an isomorphism of the homotopy groups up to dimension $n-2 k$ and it maps $\pi_{n-2 k+1}(N)$ surjectively onto $\pi_{n-2 k+1}(M)$.

\section{B. Alexandrov Spaces with Positive Curvature}

Recall that an Alexandrov space, $X$, is a finite Hausdorff dimensional complete inner metric space with a lower curvature bound in distance comparison sense (cf. Burago et al. 1992). In particular, a Riemannian manifold of sectional curvature bounded from below is an Alexandrov space. Typical examples of non-manifold type Alexandrov spaces are given by the following

Lemma 1.5 (Burago ET AL. 1992). Let $X$ be an Alexandrov space with curvature $\geq-\Lambda$. Let $G$ be a compact group of isometries. Then, the quotient space, $X / G$, is also an Alexandrov space with curvature $\geq-\Lambda$.

\section{PROOFS OF THEOREMS B AND C}

In this section we consider simply connected positively curved 6-manifolds with $G$-symmetry where $G=S O(3)$ or $S U(2)$. Note that the connected symmetry group of $S U(3) / T^{2}$ is $P S U(3)=$ $S U(3) / \mathbb{Z}_{3}$, where $\mathbb{Z}_{3}$ is the center of $S U$ (3) (cf. Shankar 2001a). It was pointed out to the author by Shankar (2001b) that the identity component of the symmetry group of the biquotient $S U(3) / / T^{2}$ is $U(2)$. In both cases, $S U(2)$ is a proper subgroup of the isometry groups. 
LEMMA 2.1. Let $M$ be a simply connected compact 6-manifold of positive sectional curvature. If $M$ admits an isometric $S U(2)$-action such that the fixed point set Fix (M, SU(2)) is not empty, then $M$ is either diffeomorphic to $S^{6}$ or $\mathbb{C P}^{3}$.

Proof. Let $X=\operatorname{Fix}(M, S U(2))$. Since an irreducible representation of $S U(2)$ has dimension 4 , the fixed point set must be of dimension 2. Therefore the orbifold $M / S U(2)$ is an Alexandrov space with boundary $X$. Thus

$$
M=E(\eta) \cup\left(S U(2) \times_{H} D^{m}\right)
$$

where $E(\eta)$ is a 4-disk bundle over $X$, and $H \subset S U(2)$ is the isotropy group for some single orbit, $m+3=\operatorname{dim} H+6$ (cf. Grove and Searle 1997). In particular, $X$ is connected, and so $X=S^{2}$ (since $X$ is totally geodesic with positive curvature). Note that the boundary of $E(\eta)$ is $S^{2} \times S^{3}$.

(i) If $H$ is of dimension 0 ;

Then the neighborhood $S U(2) \times_{H} D^{3}$ must be $S U(2) \times D^{3}$. This implies that $M$ is diffeomorphic to $S^{6}$.

(ii) If $H$ is of dimension 1 ;

Then the neighborhood $S U(2) \times{ }_{H} D^{4}$ must be $S U(2) \times{ }_{S^{1}} D^{4}$, which is equivariant diffeomorphic to $S^{2} \times D^{4}$ with the product action of $S U(2)$, i.e. on the second factor is given by the irreducible representation of $S U$ (2) on $D^{4} \subset \mathbb{C}^{2}$, on the first factor the action is given by the natural action of $S U(2)$ on $\mathbb{C P}^{1}=S^{2}$. To match the two actions on the boundary, it is easy to see that the gluing map

$$
f: S^{2} \times S^{3} \rightarrow S^{2} \times S^{3}
$$

may be written as $f(x, y)=(y \cdot x, y)$, where $y \in S^{3}=S U(2)$ acts on $x$ factoring through the natural $S O(3)$-action. It is an easy exercise to show that $M=\mathbb{C P}^{3}$.

LEMMa 2.2. Let $M$ be a simply connected compact 6-manifold of positive sectional curvature. If $M$ admits an isometric $S O(3)$-action such that the fixed point set Fix (M, SO(3)) is not empty, then $M$ is diffeomorphic to $S^{6}$.

Proof. First we claim that the fixed point set $X=\operatorname{Fix}(M, S O(3))$ must have positive dimension.

In fact, if $x \in X$ is an isolated fixed point, the local isotropy representation of $S O$ (3) gives a linear action on $S^{5}$. But a linear action of $S O(3)$ must have all principal orbit dimension 2, and so the action must have a 2-sphere as the fixed point set (cf. Bredon 1972 exercise in Chap. 5).

By the local isotropy representation we also know that $X$ has dimension 3 and so the principal orbits are of dimension 2 . Thus the orbit space is 4-dimensional with boundary $X$. Hence

$$
M=E(\nu) \cup_{\partial} S O(3) \times_{H} D^{4}
$$


where $E(v)$ is a disk bundle of dimension 3 over the fixed point set $X$, and $H$ is a circle subgroup of $S O(3)$ (cf. Grove and Searle 1994). Clearly, $X$ is a spherical form (cf. Hamilton 1982), since it is totally geodesic. In order that the boundary of the disk bundle matches the boundary of $S O(3) \times{ }_{H} D^{4}$ the core $X$ must be simply connected. By a straightforward argument one knows that $M$ is a homotopy sphere and therefore $M=S^{6}$.

The $q$-extent $x t_{q}(X), q \geq 2$, of a compact metric space $(X, d)$ is, by definition, given by the following formula:

$$
x t_{q}(X)=\left(\begin{array}{l}
q \\
2
\end{array}\right)^{-1} \max \left\{\sum_{1 \leq i<j \leq q} d\left(x_{i}, x_{j}\right):\left\{x_{i}\right\}_{i=1}^{q} \subset X\right\}
$$

Recall that the 3-extent $x t_{3}\left(S^{2}\left(\frac{1}{2}\right)\right)=\frac{\pi}{3}$ (Grove and Markvosen 1995).

LEMMA 2.3. In the orbit space $M^{*}$ there are at most three isolated singular orbits.

Proof. We prove by contradiction.

Assume that there are at least 4 isolated singular orbits. Let $A_{1}, A_{2}, \cdots, A_{4}$ be the 4 isolated singular orbits in $M^{*}$.

For any $i \neq j$, let

$$
C_{i j}=\left\{\gamma:\left[0, l_{i j}\right] \rightarrow M \mid \gamma \text { is a minimizing normal geodesic from } A_{i} \text { to } A_{j}\right\} .
$$

Let $\alpha_{i j k}=\min \left\{\measuredangle\left(\dot{\gamma}_{j}(0), \dot{\gamma}_{k}(0)\right) \mid \gamma_{j} \in C_{j i}, \gamma_{k} \in C_{j k}\right\}$. Since the curvature of $M^{*}$ is positive (cf. Lemma 1.5), by the Toponogov's comparison theorem it follows that, for each triple $(i, j, k)$ of distinct integers in $[1,4]$,

$$
\alpha_{i j k}+\alpha_{j i k}+\alpha_{i k j}>\pi
$$

Summing over all possible choices of the triple $(i, j, k)$ we know that

$$
\sum_{i=1}^{4} \sum_{1 \leq j<k \leq 4, j, k \neq i} \alpha_{i j k}>4 \pi
$$

On the other hand, for each $A_{i}$ the isotropy group $H_{i} \cong S^{1}$, note that the space of directions at $A_{i}$, $\Sigma_{A_{i}}(X)=S_{A_{i}}^{\perp} / H_{i}$, where $S_{A_{i}}^{\perp}$ is the unit sphere in the normal slice of the isolated circle orbit $A_{i}$ in $M$, which is $S^{3}(1)$. Therefore, $\Sigma_{A_{i}}(X)$ is the quotient of $S^{2}\left(\frac{1}{2}\right)$. Therefore

$$
\sum_{i=1}^{4} \sum_{1 \leq j<k \leq 4, j, k \neq i} \alpha_{i j k} \leq 4 \pi
$$

A contradiction.

Proof of Theorem B. By Lemma 2.1 we may assume that $\operatorname{Fix}(M, S U(2))$ is empty. 
By the Synge theorem the center $\mathbb{Z}_{2} \subset S U(2)$ must act on $M$ with non empty fixed point (since it preserves the orientation). Consider the fixed point set $M^{\mathbb{Z}_{2}} \subset M$. Clearly $S U$ (2) acts on $M^{\mathbb{Z}_{2}}$. If it has dimension 4, it must be $\mathbb{C P}^{2}$ or $S^{4}$ (cf. Hsiang and Kleiner 1989). But any action of $S U$ (2) on $S^{4}$ or $\mathbb{C P}^{2}$ must have fixed point. A contradiction. Therefore $M^{\mathbb{Z}_{2}}$ has dimension 2 and consists of the union of some isolated singular orbits of dimension 2. Around every singular orbit, there is a regular neighborhood of the form $S U(2) \times{ }_{H} D^{4}$ where $H$ is the isotropy group of the singular orbit (a 1-dimensional subgroup of $S U(2)$ ). In the Alexandrov space $M^{*}=M / S U(2)$, it gives a neighborhood of the singular orbit and homeomorphic to $D^{4} / H$, a cone over $S^{3} / H$. Note that the diameter of $S^{3} / H$ is at most $\frac{\pi}{2}$. By Lemma 2.3 above we know that there are at most three such isolated singular orbits.

Observe that the orbit space $M^{*}$ must be a simply connected 3-manifold (therefore $M^{*}$ is a homotopy 3-sphere) with at most three marked singular points. This shows that $M$ may be re-constructed by gluing at most three handles $S U(2) \times{ }_{H_{i}} D^{4}$ (with $H_{i}$ the isotropy groups of the singular orbits) to $S U(2) \times\left(S^{3}-\cup_{i}\right.$ int $\left.D_{i}^{3}\right)$, where $D_{i}^{3}, i=1,2,3$ are disjoint small disks neighborhood around the singular orbits in $M^{*}$. It is easy to check that the third Betti number $b_{3}(M)=0$ and, the Euler characteristic $\chi(M)=2,4$ or 6 , according to 1, 2 or 3 singular orbits.

If $\chi(M)=2$ then $M$ is homotopy equivalent to $S^{6}$ and so $M=S^{6}$ (cf. Wall 1966).

If $\chi(M)=4$ then $M=S U(2) \times_{H_{1}} D^{4} \cup_{\partial} S U(2) \times_{H_{2}} D^{4}$, where $H_{i}, i=1,2$ are circle subgroups of $S U(2)$. Since $S U(2) \times{ }_{H_{i}} \partial D^{4}=S^{2} \times S^{3}$ we get $M=S^{2} \times S^{4}$.

If $\chi(M)=6$, we now prove that $M=S U(3) / T^{2}$ or $M=S U(3) / / T^{2}$.

Let $X=S^{3}-\cup_{i=1}^{3}$ int $D_{i}^{3}$. By the above $M=S U(2) \times X \cup_{f} \cup_{i=1}^{3} S U(2) \times_{H_{i}} D^{4}$, where $f=f_{1} \cup f_{2} \cup f_{3}$ is the gluing diffeomorphism of $\cup_{i=1}^{3} S U(2) \times{ }_{H_{i}} \partial D^{4}$. Since $f$ is $S U(2)$-equivariant, each component $f_{i}$ may be identified with the diffeomorphism

$$
f_{i}: S^{2} \times S^{3} \rightarrow S^{2} \times S^{3}, \quad(x, y) \rightarrow\left(\theta_{i}(y) \cdot x, y\right)
$$

where $\theta_{i} \in \pi_{3}(S O(3)) \cong \mathbb{Z}$ is a generator and - indicates the standard action of $S O(3)$ on $S^{2}$. Observe that the topology of $M$ depends only on the isotopy class of the diffeomorphism $f$.

Fix a generator $\tau \in \pi_{3}(S O(3))$. Clearly $\theta_{i}= \pm \tau$. For simplicity we let $(+,+,+)$ denote the manifold $M$ (as well as the map $f$ ) with $f=(\tau, \tau, \tau)$. Similarly, $(-,-,-)$ denotes $M$ with $f=(-\tau,-\tau,-\tau)$, etc.

Let $h: S^{2} \times S^{3} \rightarrow S^{2} \times S^{3}$ be a diffeomorphism given by $h(x, y)=\left(x, y^{-1}\right)$, where $S^{3}=S U(2)$ is considered as a Lie group. Note that the composition of $f \circ h$ will reverse the signs, e.g. $f \circ h=(-,-,-)$ if $f=(+,+,+)$ and so the manifolds $(+,+,+)$ and $(-,-,-)$ are the same up to diffeomorphism. Therefore there are at most two different diffeomorphism types $(+,+,+)$ and $(+,-,-)$.

On the other hand, as we noted in the beginning of the section, $S U(3) / T^{2}$ and $S U(3) / / T^{2}$ both have effective isometric actions by $S U(2)$. The induced actions of $S U(2)$ on the manifolds must have only isolated singular orbits (if not, the manifold must be $S^{6}$ or $C P^{3}$ by the above argument). 
Recall that $S U(3) / T^{2}$ and $S U(3) / / T^{2}$ have different homotopy types (cf. Eschenberg 1992). Therefore $(+,+,+)$ and $(+,-,-)$ cannot be diffeomorphic. This proves the desired result.

Proof of Theorem C. By Lemma 2.2 we may assume that the fixed point set is empty.

If the principal orbit has dimension 2, since principal orbits are open dense in $M$ by the principal orbit theorem (cf. Bredon 1972), for the principal isotropy group $S^{1}$ or $O(2)$, the fixed point set of this isotropy group must be of dimension 4. Therefore $M=S^{6}$ or $\mathbb{C P}^{3}$ (cf. Grove and Searle 1994). If $S O(3) / H$ is a principal orbit type, where $H \subset S O(3)$ is a non-trivial finite group. Then the fixed point set $M^{H}$ is of dimension at least 3 for the same reason as above. Note that the fixed point set must have even codimension since $H$ preserves the orientation (cf. Kobayashi 1972). Therefore $M^{H}$ has dimension 4. By Theorem 1.5 we may conclude that $M=S^{6}$ or $\mathbb{C P}^{3}$ (cf. Fang and Rong 2001).

Now we assume that the principal orbit type is $S O(3)$.

If there is a circle subgroup $S^{1} \subset S O$ (3) with fixed point set $M^{S^{1}}$ of positive dimension, (of course it is not empty by the Synge theorem), then $\operatorname{dim} M^{S^{1}}=2$. Otherwise, $M=S^{6}$ or $\mathbb{C P}^{3}$ (cf. Grove and Searle 1994). This is impossible, since any effective $S O(3)$ action on $S^{6}$ or $\mathbb{C P}^{3}$ cannot have circle (or $O(2)$ ) isotropy group with 4-dimensional fixed point set.

Consider the minimal $S O(3)$-invariant submanifold containing $M^{S^{1}}$. It must have dimension 4 since the orbits starting from points in $M^{S^{1}}$ are all of dimension 2. In the orbit space $M^{*}=$ $M / S O(3)$, the quotient $S O(3) \cdot M^{S^{1}} / S O(3)=M^{S^{1}}$ is a boundary component. Let $\pi: M \rightarrow M^{*}$ be the quotient map. By Grove and Searle (1994) again $M^{*}$ is homeomorphic to $D^{3}$ and it contains at most one additional singular (or exceptional) orbit outside $M^{S^{1}}$ (cf. Grove and Searle 1997). Moreover,

$$
M=D(v) \cup_{\partial} S O(3) \times_{H} D^{3+\operatorname{dim} H}
$$

where $D(v)$ is a disk bundle of dimension 2 over the minimal submanifold $\pi^{-1}\left(M^{S^{1}}\right)$, and $H$ is the isotropy group of the unique orbit (it may be principal orbit).

Note that $\pi^{-1}\left(M^{S^{1}}\right)=S^{2} \times S^{2}$. The $S O(3)$ action on this $S^{2} \times S^{2}$ is equivalent to the product action of a trivial $S O(3)$ action on $S^{2}$ and the standard $S O(3)$ on $S^{2}$ (with just one orbit).

If $H$ is a finite group, then $H=\{1\}$ and so $M=\mathbb{C P}^{3}$. Indeed, in this case the action is equivalent to the one defined by: $A \cdot\left[z_{0}, z_{1}, z_{2}, z_{3}\right]=\left[A\left(z_{0}, z_{1}\right), A\left(z_{2}, z_{3}\right)\right]$, where $\left[z_{0}, z_{1}, z_{2}, z_{3}\right]$ is the homogeneous coordinate and $A \in S U(2) / \mathbb{Z}_{2}=S O(3)$.

We now prove that $\operatorname{dim} H=0$. In fact, since $v$ is a $S O$ (3)-equivariant oriented 2-bundle over $S^{2} \times S^{2}$. Using standard algebraic topology we know that the Euler class $e(v)$ has to be even. If $H$ has positive dimension, it must be $S^{1}$ or $O(2)$. Since all other orbits are principal, $H$ must be $S^{1}$ and so $S O(3) \times{ }_{H} D^{4}=S^{2} \times D^{4}$. This is impossible since $e(v)=0(\bmod 2)$ implies that boundary $S O(3) \times{ }_{H} \partial D^{4}$ has fundamental group at least $\mathbb{Z}_{2}$.

Finally we consider the case of $M^{S^{1}}$ is zero dimensional for all circle subgroup in $S O(3)$. In other words, singular orbits are isolated. By Lemma 2.3 once again we know that there are at most 3 
isolated singular orbits. As in the proof of Theorem B, the possible values of $\chi(M)=2,4,6$. In the case $\chi(M)=4$ one has $M=S O(3) \times{ }_{H_{1}} D^{4} \cup_{\partial} S O(3) \times{ }_{H_{2}} D^{4}=S^{2} \times S^{4}$, up to diffeomorphism. We claim that $\chi(M)=2$ and 6 are impossible. This will complete the proof.

Let $X=M^{*}-\cup_{i=1}^{l}$ int $D_{i}^{3}$ with $l$ the number of singular orbits (here $l=1$ or 3 ), where $D_{i}^{3}$ is as in Theorem A. Note that $M^{*}$ is again a homotopy 3-sphere. Since all orbits in $X$ is of type $S O(3)$, the principal $S O(3)$-action on $\pi^{-1}(X)$ corresponds to a unique classifying map $f: X \rightarrow B S O$ (3) for the action. Note that $f$ restricts on each piece of the boundary (a 2-sphere) of $X$ gives the classifying map of the free $S O(3)$ action on $S O(3) \times{ }_{H_{i}} S^{3}=S^{2} \times S^{3}$, which is not null homotopic in $\pi_{2}(B S O(3))=\mathbb{Z}_{2}$. Clearly, the sum of the $l$ boundary pieces must be zero homologous (since the map $f$ is a cycle with boundary the sum). Therefore $l$ must be even. This proves that there are only 2 singular orbits. This proves the desired result.

\section{ACKNOWLEDGMENTS}

This paper is written based on the author's talks in Brazilian Colloquium in IMPA and in the conference "Foliation and Geometry 2001" in PUC, Brazil. Supported by CNPq of Brazil, NSFC Grant 19741002, RFDP and Qiu-Shi Foundation of China.

\section{RESUMO}

Seja $M$ uma 6-variedade compacta, simplesmente conexa de curvatura sectional positiva. Se a identidade componente do grupo de isometria contém um subgrupo de Lie simples, provamos que $M$ é difeomórfico de uma das 5-variedades listadas no Teorema A.

Palavras-chave: 6-variedades, curvatura positiva, grupos de simetria.

\section{REFERENCES}

AlofF S AND Wallach NR. 1975. An infinite family of 7-manifolds admitting positive curved Riemannian structures. Bull Amer Math Soc 81: 93-97.

Berger M. 1961. Les Variétés Riemanniennes homogènes normales simplement connexes à courbure strictement positive. Ann Scuola Norm Sup Pisa 15: 179-246.

BERGERy LB. 1976. Les variétés Riemanniennes homogènes simplement connexes de dimension impair à courture strictement positive J Math Pures App 155: 47-68.

BREDON G. 1972. Introduction to compact transformation groups, vol 48. Academic Press.

Burago Y, Gromov M and Perel'man AD. 1992. Alexandrov spaces with curvature bounded below. Uspekhi Mat Nauk 47(2): 3-51.

Eschenberg J-H. 1982. New examples of manifolds with strictly positive curvature. Invent Math 66: 469-480.

EsCHENBERG J-H. 1992. Inhomogeneous spaces of positive curvature, Differential Geom Appl 2: 123-132. 
FAng F And Rong X. 2001. Positively curved manifolds of maximal discrete symmetry rank. Preprint (revised version).

FANG F AND Rong X. 2002. Topology of positively curved manifolds of almost maximal symmetry rank. To appear.

FAng F, Mendonça S AND Rong X. 2002. Connectedness principles in the geometry of positive curvature. Preprint (revised version).

Grove K and Markvosen S. 1995. New extremal problems for the Riemannian recognition program via Alexandrov geometry. J Amer Math Soc 8: 1-28.

Grove K and Searle C. 1994. Positively curved manifolds with maximal symmetry-rank. J Pure Appl Alg 91: 137-142.

Grove K and Searle C. 1997. Differential topological restrictions curvature and symmetry. J Diff Geom 47: $530-559$.

Hamilton R. 1982. Three-manifolds with positive Ricci curvature. J Diff Geom 17: 255-306.

Hsiang W. 1975. Cohomology theory of topological transformation groups. Ergebnisse der Mathematik und inere Grenzgebiete, Springer-Verlag.

HSIANG W AND KLEINER B. 1989. On the topology of positively curved 4-manifolds with symmetry. J Diff Geom 30: 615-621.

KoваYASHi S. 1972. Transformation groups in differential geometry. Springer-Verlag Berlin Heidelberg New York.

RoNG X. 2002. Positively curved manifolds with almost maximal symmetry rank. Geometriae Dedicata. To appear.

SHANKAR K. 2001a. Isometry groups of homogeneous spaces with positive sectional curvature. Diff Geom Appl 14: 57-78.

ShanKar K. 2001b. Private communication.

WaLl CTC. 1966. Classification problems in differential topology. V. On certain 6-manifolds. Invent Math 1: 355-374.

WILKING B. 2002. In preparation. 